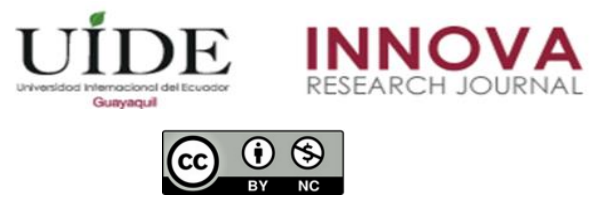

INNOVA Research Journal, ISSN 2477-9024

(Septiembre-Diciembre 2020). Vol. 5, No.3.1 pp. 315-328

DOI: https://doi.org/10.33890/innova.v5.n3.1.2020.1544

URL: http://revistas.uide.edu.ec/index.php/innova/index

Correo: innova@uide.edu.ec

\title{
Test Minimental para el diagnóstico temprano del deterioro cognitivo
}

\section{Minimental test for early diagnosis of cognitive impairme}

Jhonatan Gualberto Llamuca Quinaloa

https://orcid.org/0000-0002-9676-8449

Yessenia Karolina Macías Guamangate

https://orcid.org/0000-0002-0422-6731

José Leonardo Miranda Caisaluisa

https://orcid.org/0000-0001-9799-5283

Verónica Del Consuelo Tapia Cerda

(D) https://orcid.org/0000-0002-5338-7814

Universidad Técnica de Cotopaxi, Ecuador

Autor para correspondencia: jhonatan.1lamuca5258@utc.edu.ec;

yessenia.macias6708@utc.edu.ec; jose.miranda7348@utc.edu.ec; veronica.tapia@utc.edu.ec;

Fecha de recepción: 18 de septiembre de 2020 - Fecha de aceptación: 21 de noviembre de 2020

\section{Resumen}

Este trabajo informa el proceso y resultados iniciales de la automatización del test de diagnóstico médico de atención primaria en salud, estandarizado y reconocido a nivel mundial para la detección temprana del deterioro cognitivo en pacientes geriátricos denominado Test MiniMental. Es un cuestionario que evalúa la memoria, orientación, concentración y lenguaje; se basa en el otorgamiento de puntuaciones según las respuestas del paciente, luego las puntuaciones se promedian y se comparan con los parámetros estandarizados en el test determinando de esta manera el nivel de deterioro cognitivo que puede sugerir o no el inicio de patologías psiquiátricas como el Alzheimer. La aplicación creada permite seguir de forma secuencial y gráfica la evolución clínica del paciente; incluye también un componente de recordatorios que avisa al paciente la medicación a tomar según prescripción médica indicada. Para validar el trabajo se contó con la colaboración del médico ocupacional de la Universidad Técnica de Cotopaxi y la psicóloga clínica del Hogar de Ancianos Instituto Estupiñán de Latacunga - Ecuador, institución donde se aplicó la herramienta a 12 pacientes de 75 a 93 años, 5 varones y 7 mujeres, como resultado se identificó deterioro cognitivo grave en 4 pacientes, deterioro cognitivo entre discreto y moderado en 6 pacientes.

Palabras claves: herramienta informática; deterioro cognitivo, pacientes geriátricos; detección temprana.

\begin{abstract}
This work informs the initial process and results of the automation of the medical diagnostic test of primary health care, standardized and recognized worldwide for the early detection of cognitive impairment in geriatric patients called MiniMental Test. It is a questionnaire that evaluates
\end{abstract}


memory, orientation, concentration and language; is based on a score given to the patient's responses, then the scores are averaged and compared to the standardized parameters in the test, determining the level of cognitive impairment that may or may not suggest the onset of psychiatric pathologies such as Alzheimer's. The application created allows to follow sequentially and graphically the clinical evolution of the patient; it also includes a reminder component that alerts the patient to the medication to be taken according to the indicated medical prescription. The validation of this work was carried out by the occupational physician at Cotopaxi Technical University of Cotopaxi and the clinical psychologist of the Home of the Elderly "Instituto Estupiñán" of Latacunga - Ecuador, institution where the tool was applied to 12 patients aged 75 to 93 years, 5 males and 7 women, as a result severe cognitive decline was identified in 4 patients, cognitive impairment between discrete and moderate in 6 patients.

Keywords: computer tool; cognitive impairment; geriatric patients; early detection.

\section{Introducción}

En el 2013, se estimó que más de 44 millones de personas vivían con demencia en todo el mundo y se predice la prevalencia a duplicarse cada 20 años, alcanzando los 76 millones en 2030 (Rodríguez y Gutiérrez Herrera, 2014) un Informe Mundial de Alzheimer publicado en el año 2016 estima que en el 2015 ya existían más de 46.8 millones de personas que viven con demencia, (Polzer y Gewald, 2017) la misma que en el 2010 causó aproximadamente 486,000 muertes (Pirani, Bulakiwal, Kagalwala, y Kalolwala, 2016). El costo socioeconómico mundial de la demencia fue de US \$ 604. Miles de millones en 2010, (Rodríguez y Gutiérrez Herrera, 2014) Estados Unidos gastó en el 2016 más de \$ 236 mil millones de dólares en la enfermedad, creando una carga desalentadora. Considerando que no hay tratamientos curativos disponibles para la demencia. (Imtiaz, Khan, y Seelye, 2018).

El deterioro cognitivo puede ser causa de enfermedades como al Alzheimer que en términos médicos implica un padecimiento cerebral degenerativo (Violetta y Susana, 2019) en algunos países de Europa y en Estados Unidos esta enfermedad es considerada un problema de salud pública que afecta por lo general a la población de adultos mayores, convirtiéndolos en un peso para la familia y la sociedad.

Según (Dallas, Calvin, Hailey, Sudeep, y Nathan, 2018) la enfermedad de Alzheimer y otras formas de demencia son cada vez más comunes con el envejecimiento de la mayoría de las poblaciones. La mayoría de las personas con demencia se presentarán primero para recibir atención y evaluación en entornos de atención primaria. Existe la necesidad de instrumentos breves de detección de demencia que puedan diagnosticar con precisión la demencia en entornos de atención primaria.

El Mini-Mental State Examination (MMSE) es una prueba escrita que se utiliza habitualmente como parte del proceso cuando se considera un diagnóstico de demencia, con una puntuación máxima de 30, en la que las puntuaciones inferiores indican problemas cognitivos más graves. Es una de las herramientas más utilizada a nivel mundial por su brevedad y fácil aplicación (Tombaugh y Mcintyre, 1992), cuenta con 10 áreas de valoración: orientación espaciotemporal, registro de tres palabras, fijación de atención, memoria, nominación verbal, 
repetición y comprensión, lectura, escritura y construcción viso espacial, (Mora Villalobos, y otros, 2017). El punto de corte establecido para el MMSE define la función cognitiva "normal" y generalmente se fija en 24, aunque teóricamente podría estar en cualquier lugar entre 1 y 30 (Arevalo-Rodriguez I, 2016).

La demencia es un síndrome de deterioro cognitivo global progresivo. En 2010, se estimaba que más de 35 millones de personas en todo el mundo vivían con demencia. Algunas personas con deterioro cognitivo leve (DCL) progresarán a demencia, pero otras se mantendrán estables o recuperarán su función completa. Existe un gran interés en encontrar buenos predictores de demencia en personas con DCL. El Mini Examen del Estado Mental (MMSE) es la herramienta de detección breve más conocida y más utilizada para proporcionar una medida general del deterioro cognitivo en entornos clínicos, de investigación y comunitarios (ArevaloRodriguez I, 2016).

Varios estudios han demostrado que la telemedicina es un vehículo para la información y el apoyo, ya sea a pedido o como parte de un programa de seguimiento, que mejora la calidad y optimización del tratamiento (Alliance, 2017).

En el caso de la herramienta informática que se propone, automatiza el test MMSE, de tal manera que se pueda obtener de forma rápida y exacta los resultados de la prueba con el nivel de deterioro cognitivo del paciente. Está desarrollada en el lenguaje de programación de alto nivel Java con el servidor de base de datos PostgreSQL, está pensada en tres perfiles de usuario: terapeuta, paciente, administrador; ésta primera versión es de tipo escritorio, pero se van a desarrollar próximas versiones, perfeccionando y ampliando la funcionalidad de evaluación a otros métodos, mejorando flexibilidad y uso, en otras arquitecturas como web y móvil.

\section{Desarrollo de la aplicación}

a) Estructura del sistema

La Figura 1 presenta una visión conceptual de la estructura de la aplicación desarrollada, como se aprecia, tiene 4 módulos funcionales que son: Evaluar, Recordatorio de medicamentos, Ejercicios mentales y Guía educativa. 


\section{Figura 1}

\section{Modelo conceptual del sistema}

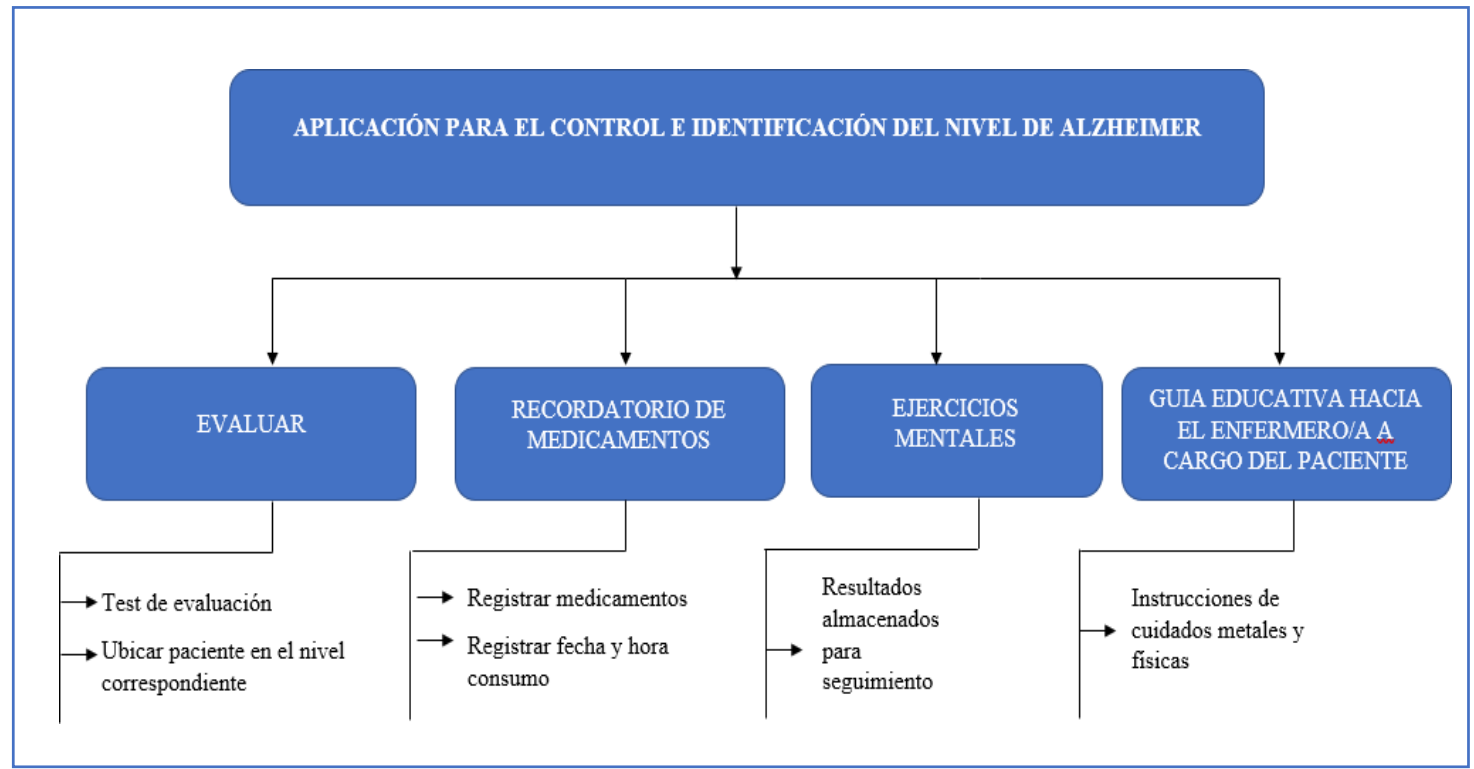

b) Modelo de desarrollo Iterativo - Incremental

El modelo de procesos utilizado para implementar la herramienta informática es el incremental, éste combina elementos de los flujos del proceso lineal y paralelo; aplica secuencias lineales (iteraciones) en forma escalonada a medida que avanza el calendario de actividades, en cada iteración se produce un incremento de software que pueden ser completamente funcional y por lo tanto entregable (S., 2010).

Bajo el enfoque de este modelo se construyó la aplicación, para lo cual se fraccionó la construcción de la solución en 4 iteraciones en las que se ejecutaron de manera secuencial las etapas del modelo prescriptivo lineal o clásico, denominado en cascada (análisis, diseño, implementación, pruebas).

\section{Análisis de requerimientos}

En la Tabla. 1 se muestran las 4 iteraciones, cada una con requerimientos específicos a implementar, el tiempo de desarrollo estuvo estimado en tiempos de 3 a 4 semanas, de tal manera que sea fácil la detección de errores y la obtención de retroalimentación del usuario. 


\section{Tabla 1}

Iteraciones a desarrollar

\begin{tabular}{|c|c|}
\hline Iteración & Requisito Funcional (RQ) \\
\hline 1 & $\begin{array}{l}\text { RF001 (Evaluar Paciente) } \\
\text { RF002 (Elegir tipo de paciente) } \\
\text { RF003 (Registrar información) } \\
\text { RF004 (Registrar medicamentos) }\end{array}$ \\
\hline 2 & $\begin{array}{l}\text { RF005 (Generar alarma) } \\
\text { RF006 (Ejercicios mentales) }\end{array}$ \\
\hline 3 & $\begin{array}{l}\text { RF007 (Guía Educativa) } \\
\text { RF008 (Registro contacto) } \\
\text { RF009 (Eliminar contacto) }\end{array}$ \\
\hline 4 & $\begin{array}{l}\text { RF0010 (Editar medicamentos) } \\
\text { RF0011 (Eliminar medicamentos) } \\
\text { RF0012 (Actualizar contactos) }\end{array}$ \\
\hline
\end{tabular}

Fuente: elaboración propia

La técnica de análisis utilizada es la técnica de Casos de Uso, a continuación, la figura 2 y la tabla 2 respectivamente, ilustran el diagrama de casos de uso y el caso de uso extendido del requisito Evaluar Paciente.

Figura 2

Diagrama de casos de uso "Evaluar paciente",

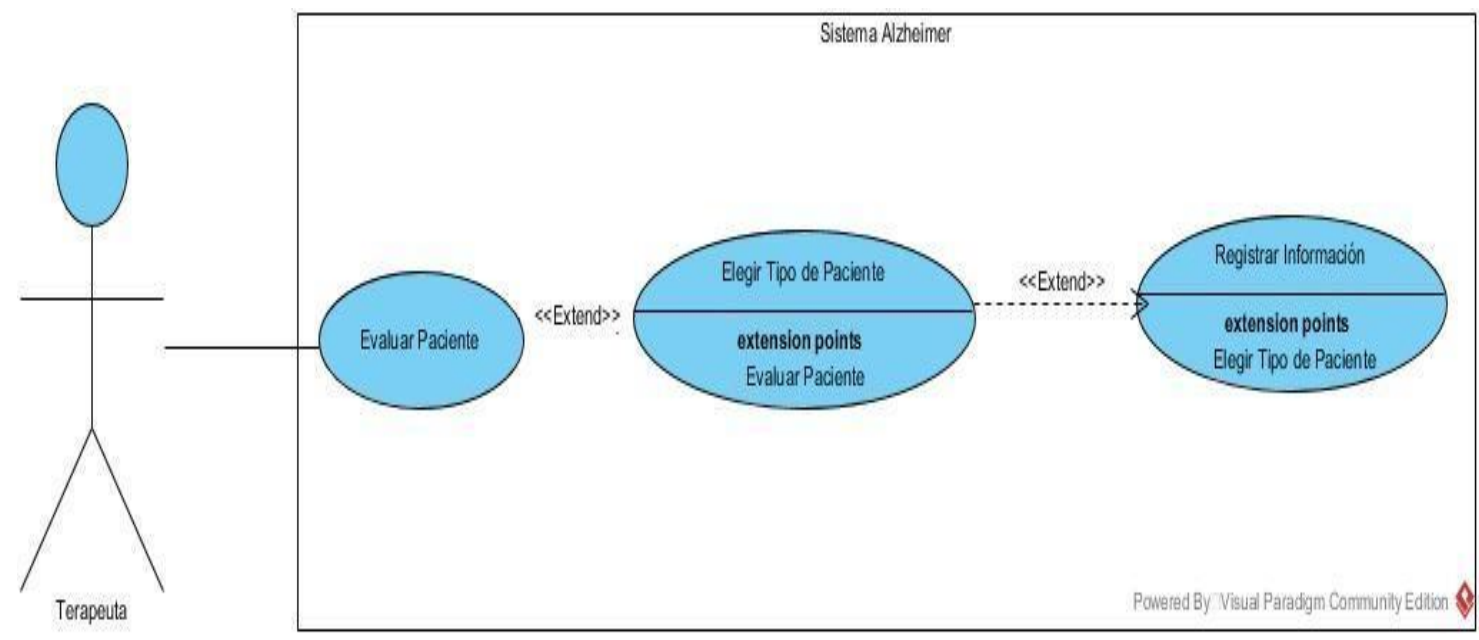




\section{Tabla 2}

Caso de uso extendido "Evaluar Paciente"

$$
\mathrm{N}^{\circ} \quad \text { CU001 }
$$

Nombre: Evaluar Paciente

Autor: Llamuca Jhonatan Macías Yessenia Miranda Leonardo

\section{Fecha: $\quad 27 / 09 / 2019$}

\section{Descripción:}

Caso de uso que permite al terapeuta evaluar al paciente haciendo uso del test Mini-Mental.

\section{Actores:}

Terapeuta.

\section{Precondiciones:}

El terapeuta debe haber ingresado al sistema.

\section{Flujo Normal:}

1.El terapeuta selecciona la opción Test de Evaluación.

2. El sistema muestra la interfaz con el del test de evaluación.

3. El terapeuta selecciona intervalo de edad del paciente.

4. El terapeuta selecciona cada una de las respuestas dadas por el paciente, presiona Obtener Calificación.

5. El sistema muestra la puntuación obtenida por el paciente.

6. El terapeuta presiona Finalizar Test.

7. El sistema muestra una ventana con la puntuación y el nivel de Alzheimer del paciente.

\section{Flujo Alternativo:}

6.1Se debe presionar un intervalo de edad caso contrario no se finaliza el test y regresa al paso 2.

6.2Se debe presionar Obtener Calificación caso contrario no se finaliza el test y regresa al paso 2.

\section{Poscondiciones:}

El terapeuta evalúa al paciente.

Fuente: elaboración propia

\section{Diseño}

En la fase de diseño se realiza el modelado del sistema a través del Lenguaje de Modelado Unificado (UML) (Erik F. Méndez Garcés, 2019), la figura 3 presenta el diagrama de actividad del caso de uso "Evaluar Paciente"; $y$, la figura 4 el diagrama de secuencias del mismo caso de uso. 


\section{Figura 3}

Diagrama de actividad 'Evaluar paciente,'

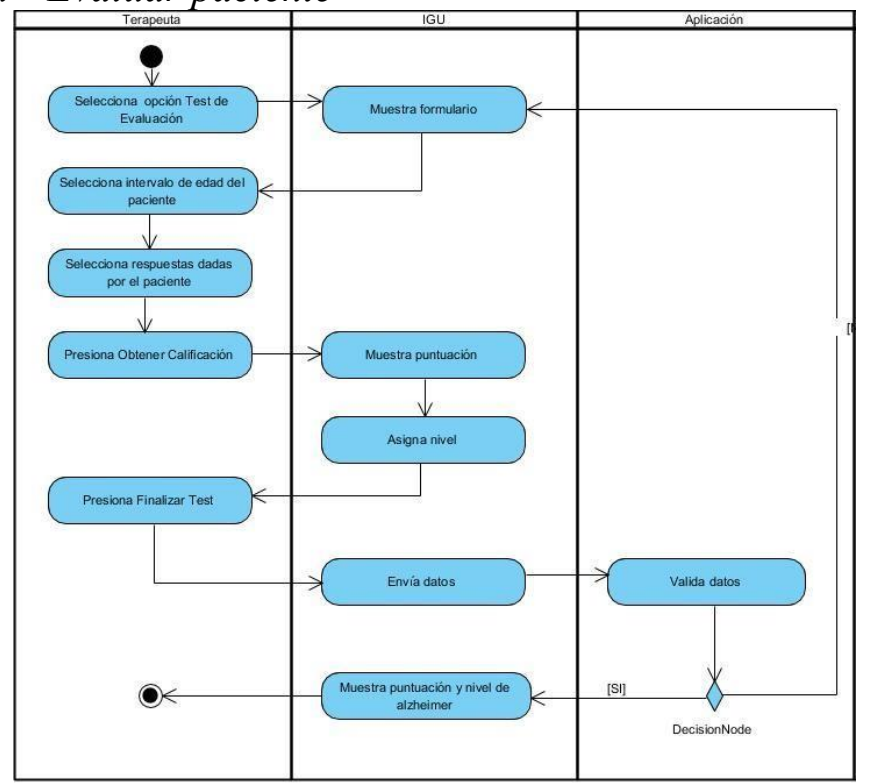

Figura 4

Diagrama de secuencias "Evaluar paciente"

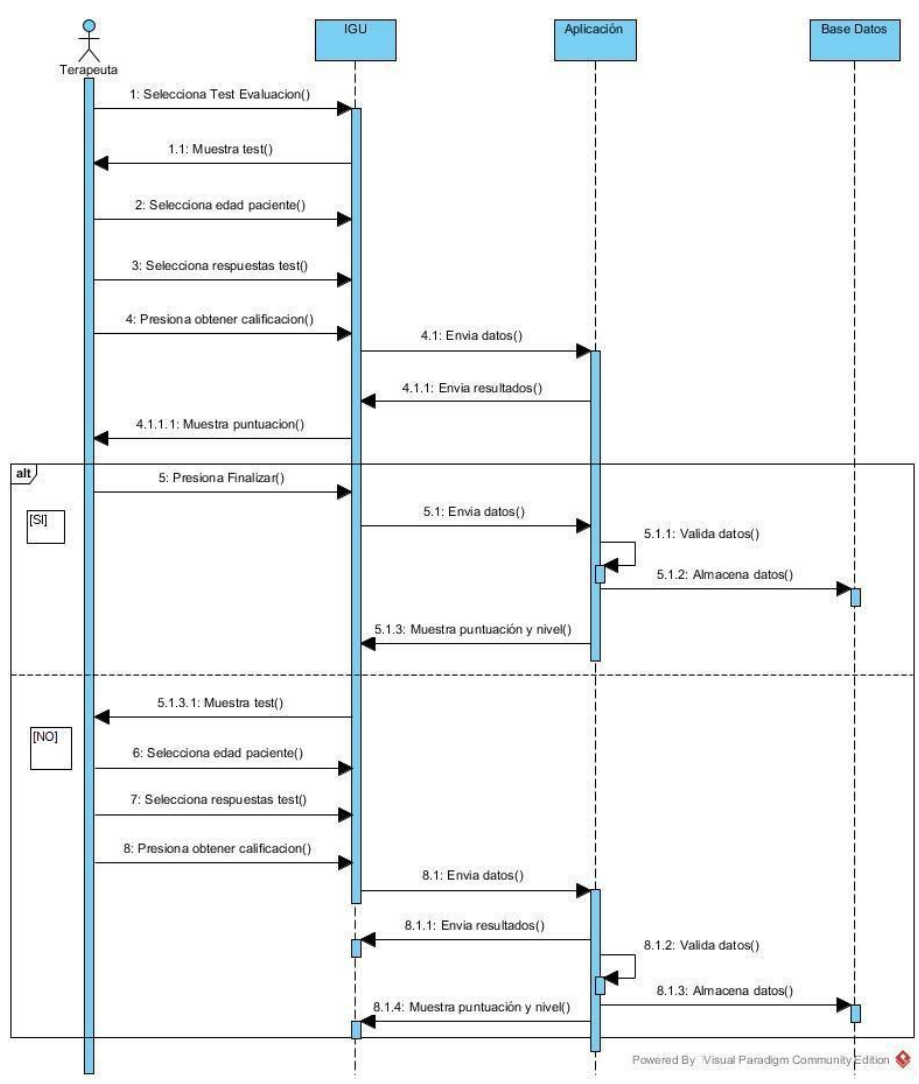

Esta obra se comparte bajo la licencia Creative Common Atribución-No Comercial 4.0 International (CC BY-NC 4.0) Revista de la Universidad Internacional del Ecuador. URL: https://www.uide.edu.ec/ 


\section{Implementación}

En la fase de implementación se codifica cada uno requisitos funcionales, la figura 4 presenta una muestra del código desarrollado para el caso de uso "Ejercicios mentales". Así mismo, las figuras 5 y 6, muestran las interfaces gráficas de usuario (IGU) de los casos "Ejercicios mentales" y "Evaluar paciente", respectivamente.

\section{Figura 5}

\section{Código 'Ejercicios mentales'”}

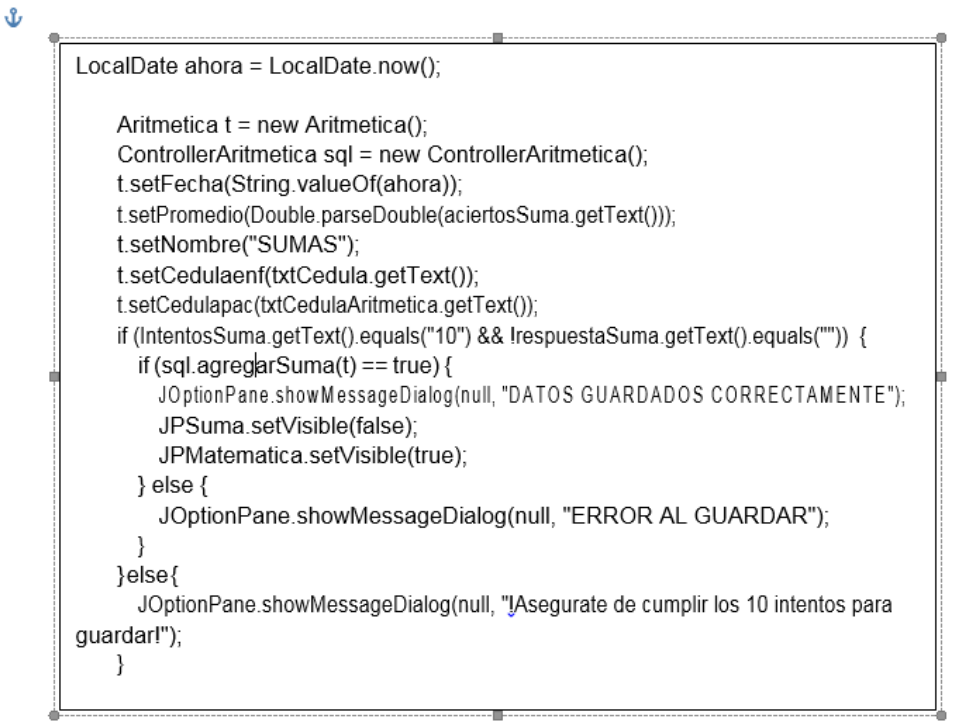

\section{Figura 6}

$I G U$, 'Ejercicios mentales",

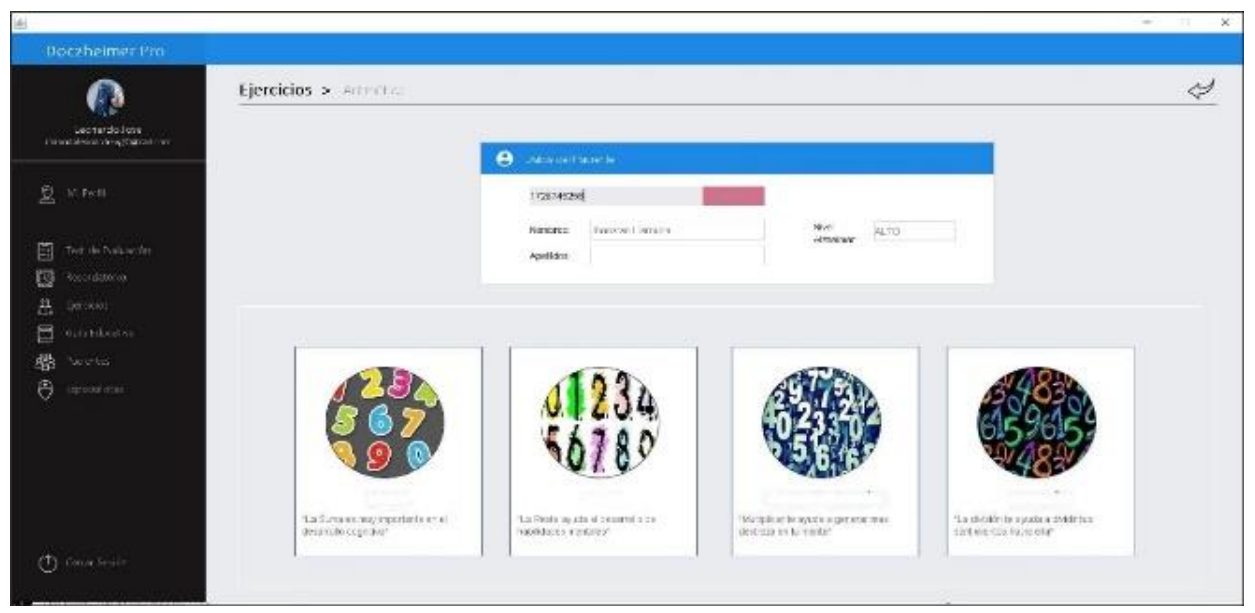




\section{Figura 7}

$I G U$, 'Evaluar paciente",

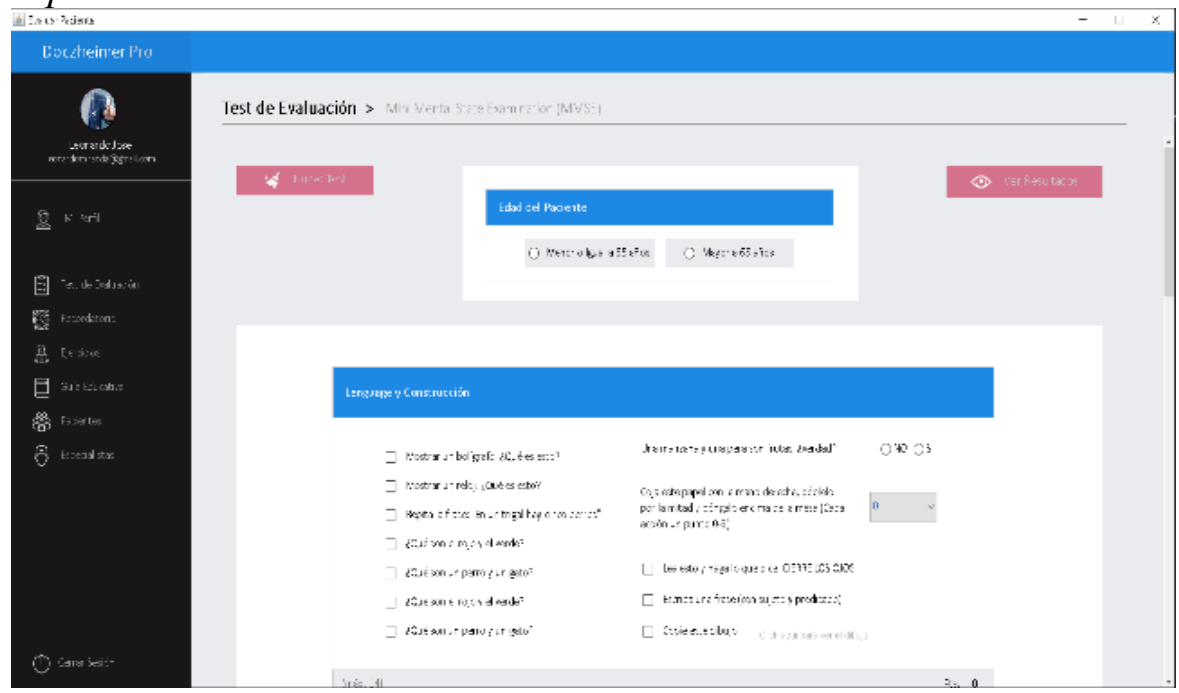

Pruebas

En esta fase se ejecutaron pruebas funcionales de cada requerimiento a través de casos de prueba, el objetivo es identificar fallos en el sistema, producidos por errores de programación. En la Tabla 3, se presenta un ejemplo de los casos de prueba para "Ejercicios mentales".

\section{Tabla 3}

Casos de prueba 'Ejercicios mentales"'

\begin{tabular}{|c|c|c|}
\hline $\begin{array}{l}\text { Número de } \\
\text { Caso }\end{array}$ & Datos de entrada & Resultados Esperados \\
\hline CP001 & Null, 0201912517 & $\begin{array}{l}\text { Para acceder a un ejercicio, es necesario primero } \\
\text { buscar al paciente. } \\
\text { Si el campo cédula está vacío y se realiza la } \\
\text { búsqueda, genera el mensaje de error } \\
\text { 'PRIMERO BUSCA A UN PACIENTE'. } \\
\text { Si el paciente no se encuentra registrado genera el } \\
\text { mensaje de error, 'USUARIO NO SE } \\
\text { ENCUENTRA REGISTRADO'. }\end{array}$ \\
\hline $\begin{array}{l}\text { CP002 } \\
\text { (Lecturas) }\end{array}$ & Cuestionario & $\begin{array}{l}\text { Si intenta guardar la puntuación del test, y el } \\
\text { promedio aún no está generado, se genera el mensaje } \\
\text { de error 'PRIMERO FINALIZA EL } \\
\text { CUESTIONARIO PARA GUARDARLO'. }\end{array}$ \\
\hline $\begin{array}{l}\mathrm{CP} 003 \\
\text { (Aritmética) }\end{array}$ & $\begin{array}{l}\text { Sumas/Restas/Multiplica } \\
\text { ción/División }\end{array}$ & $\begin{array}{l}\text { Son } 10 \text { intentos, si no se culmina un intento, y se } \\
\text { intenta continuar al siguiente, genera el mensaje de }\end{array}$ \\
\hline
\end{tabular}




\begin{tabular}{|c|c|}
\hline $\begin{array}{l}\text { Número de Datos de entrada } \\
\text { Caso }\end{array}$ & Resultados Esperados \\
\hline & $\begin{array}{l}\text { error 'ASEGURATE DE CUMPLIR PRIMERO EL } \\
\text { INTENTO'. } \\
\text { Si no se cumplen los } 10 \text { intentos y se quiere guardar } \\
\text { la puntuación obtenida, genera el mensaje de error } \\
\text { 'ASEGURATE DE CUMPLIR LOS } 10 \\
\text { INTENTOS PARA GUARDAR'. }\end{array}$ \\
\hline
\end{tabular}

Fuente: Elaboración propia

Con los casos de prueba, se ejecuta cada funcionalidad, controlando que los resultados esperados sucedan de acuerdo a lo planificado.

c) Despliegue y Validación

El sistema fue puesto en producción en el Hogar de Ancianos Instituto Estupiñán Latacunga, ubicado en la ciudad de Latacunga - Ecuador, esta institución tiene como objetivo albergar y acoger a adultos mayores desvalidos y sin referencia familiar, en la actualidad atiende integralmente a alrededor de 65 adultos mayores con los servicios de gerontología, nutrición, psicología, fisioterapia y cuidado personal (La Hora Cotopaxi, 2018).

Se realizó la evaluación a 23 pacientes del asilo, se contó con la colaboración de la psicóloga de la institución y en la interpretación médica de los resultados obtenidos, intervino el responsable del Departamento Médico Ocupacional de la Universidad Técnica de Cotopaxi, Dr. Richard Pérez Hidalgo. La primera evaluación se realizó el 21 de junio del 2019 a las 23 personas; posteriormente para el seguimiento, se trabajó con 12 pacientes, consideradas residentes permanentes de la institución; se realizó una segunda evaluación el 18 de diciembre del 2019 y una tercera el 04 de febrero del 2020.

A continuación, en la figura 8 se visualiza, un ejemplo del reporte gráfico de seguimiento que otorga la herramienta, cabe mencionar que se oculta el nombre del paciente por el derecho de confidencialidad estipulado en el Código Orgánico de la Salud de Ecuador (CONASA, 2018). 


\section{Figura 8}

Reporte gráfico de la evaluación del paciente $N N$ de 77 años

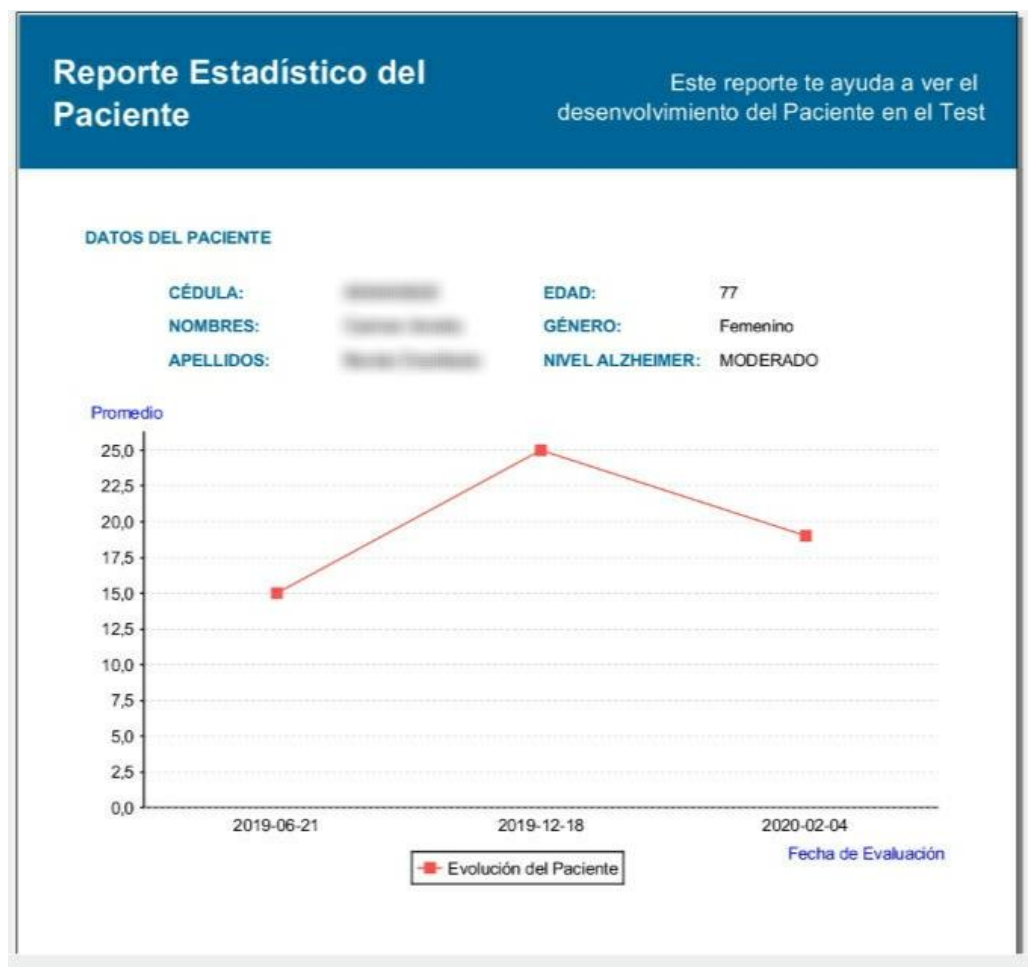

\section{Resultados y discusión}

En esta sección se detallan los resultados obtenidos con la aplicación a la que se le ha denominado MINIMENTOOL V1.0. Esta herramienta informática como ya se ha mencionado en secciones anteriores, automatiza el instrumento Minimental para la evaluación breve del estado mental, sobre todo en ancianos; investiga cinco dominios cognitivos que son esencialmente: orientación, fijación, concentración y cálculo, memoria y lenguaje. (Llamas Velasco Sara, 2015) (E, 2016).

MINIMENTOOL se aplicó a 12 pacientes, de entre 75 y 93 años, de los cuáles 5 son varones y 7 mujeres. El estudio se realizó durante las primeras horas de la mañana con la mayor comodidad posible para los pacientes; todos los participantes del estudio tenían un nivel de escolaridad media de ciclo básico.

En cuanto a los resultados obtenidos, se observó el mayor deterioro en la valoración del nivel de concentración; un deterioro medio en la valoración del nivel de memoria y orientación; siendo el nivel menos deteriorado en la valoración, el del lenguaje y la fijación; basados en el promedio de respuestas de los pacientes. Figura 9. 


\section{Figura 9}

Niveles de deterioro

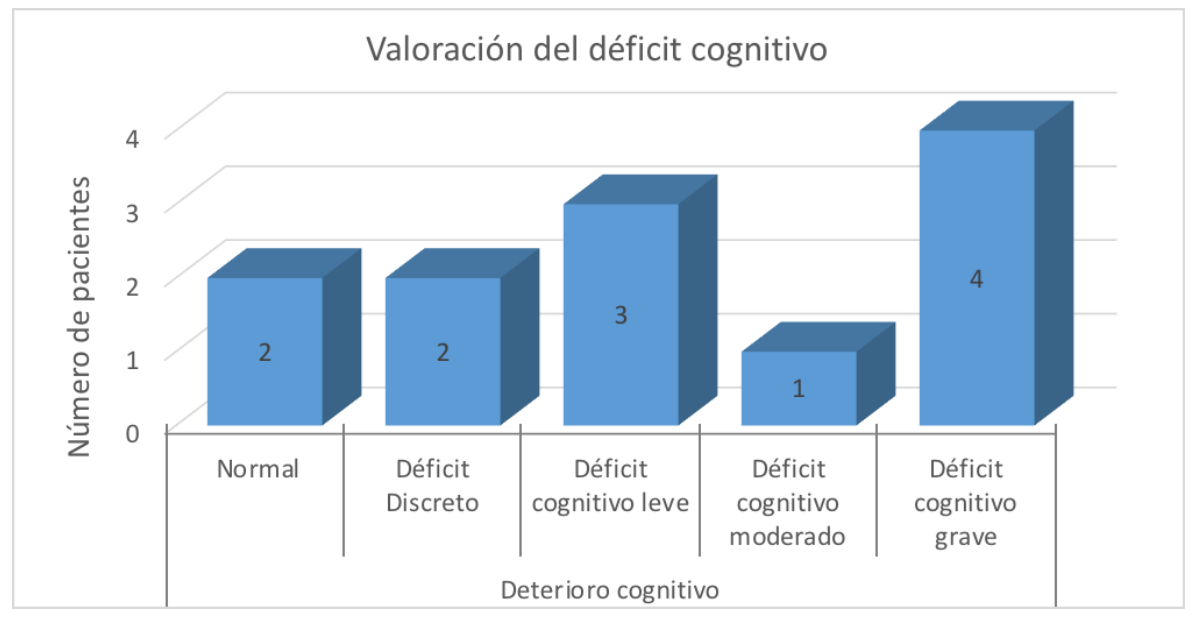

La herramienta informática, MINIMENTOOL, se utilizó para el seguimiento y documentación del nivel de deterioro cognitivo de estos 12 pacientes, observándose a 2 de ellos con un nivel cognitivo normal; 2 con déficit discreto; 3 con déficit cognitivo leve; 1 con déficit cognitivo moderado y 4 con déficit cognitivo grave.

En la ilustración 10, se ilustra el puntaje promedio obtenido en la evaluación de cada nivel cognitivo comparado con el puntaje asignado por el instrumento, para poder determinar en qué nivel se presenta el mayor deterioro cognitivo. Como se puede observar, la concentración presenta el mayor déficit cognitivo en los 12 pacientes estudiados.

\section{Figura 10}

Comparación de los valores promedio con el valor de referencia

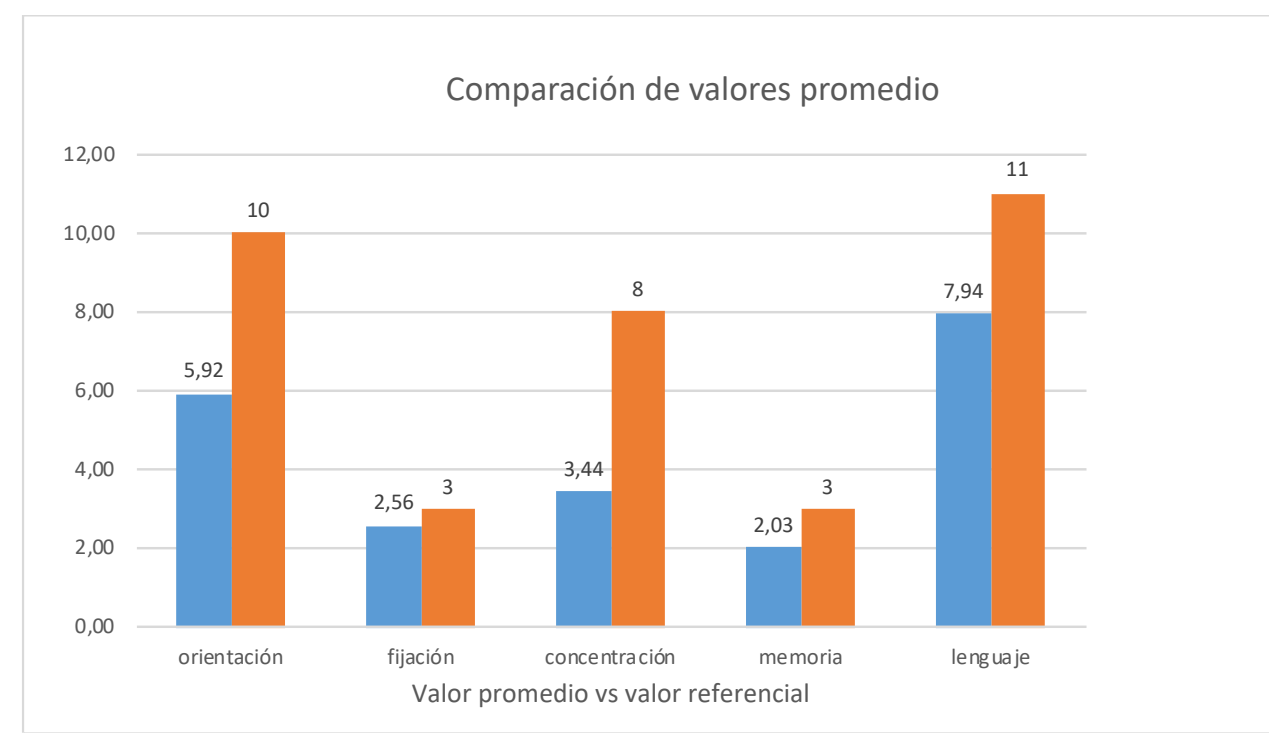




\section{Conclusiones}

Es importante que la tecnología facilite el uso de herramientas clínicas preexistentes, buscando siempre ampliar la visión médica en la atención primaria en salud. Minimental como instrumento diagnóstico del deterioro cognitivo se sigue constituyendo en una forma rápida y segura de valoración en el nivel primario de salud; que permite al profesional de la salud de ese nivel de atención, derivar de forma documentada y adecuada a un paciente a un nivel de mayor complejidad diagnóstica o de especialidad. Con esta herramienta informática se logra obtener estadísticas confiables para el seguimiento del nivel cognitivo en pacientes geriátricos, lo hace de manera automática y eficiente; así lo demuestran los resultados obtenidos en las pruebas de validación realizadas a 12 pacientes de la tercera edad, a quienes se les aplicó la herramienta determinando en 10 de ellos déficit de cognición; esta validación realizada con la vigilancia de expertos en el área de salud, permite concluir que se ha conseguido el objetivo trazado, pues MINIMENTOOL es una aplicación informática creada a través del uso de técnicas e instrumentos de ingeniería de software que permite diagnosticar los niveles cognitivos de un paciente geriátrico para la detección temprana de enfermedades mentales como el Alzheimer.

\section{Recomendaciones}

Se plantea nuevos trabajos que extiendan el alcance del trabajo presentado en esta ocasión, una siguiente versión de la aplicación para dispositivos móviles, de tal manera que los usuarios puedan manejar MINIMENTOOL a través de sus teléfonos inteligentes con nuevas funcionalidades como notificaciones de horario de medicamentos, calendario de citas médicas, entre otras. Esta nueva versión podría convertirse en una herramienta de apoyo para los familiares y cuidadores de los adultos mayores que deben estar pendientes del deterioro cognitivo que se pueda presentar en ellos, con el fin de buscar atención profesional especializada en etapas tempranas a partir de la detección de problemas de cognición.

\section{Agradecimientos}

A la Universidad Técnica de Cotopaxi y al Hogar de Ancianos Instituto Estupiñán Latacunga, por apoyar la ejecución de este proyecto y permitir su validación como aporte a la sociedad, a la medicina preventiva y al desarrollo tecnológico.

\section{Referencias Bibliográficas}

Alliance, F. C. (2017). Family Caregiver Alliance. Obtenido de Family Caregiver Alliance: https://www.caregiver.org/alzheimers-disease-caregiving

Arevalo-Rodriguez I, S. N.-P. (2016). Mini-Mental State Examination (MMSE) para la detección de la demencia en las personas de 65 años o mayores. Cochrane(CD011145).

CONASA, C. N. (2018). Código Orgánico de Salud. Quito. 
Dallas, S., Calvin, C., Hailey, N., Sudeep, G., \& Nathan, H. (2018). Mini-Cog for the diagnosis of Alzheimer's disease dementia and other dementias within a primary care setting. Cochrane Systematic Review - Diagnostic .

E, G. O. (noviembre de 2016). Infogerontología. Recuperado el 20 de octubre de 2019, de www.inforgerontologia.com

Erik F. Méndez Garcés, G. M. (2019). Analysis, Review and Development of a Conceptual Model, based on Class Diagrams as a Component of UML, Focused on Industrial Automation. International Journal of Control Systems and Robotics (4).

Imtiaz, D., Khan, A., \& Seelye, A. (2018). A Mobile Multimedia Reminiscence Therapy. Hindawi, 10.

La Hora Cotopaxi. (12 de JULIO de 2018). El Asilo de Latacunga abre sus puertas a la ciudadanía. Diario la Hora.

Llamas Velasco Sara, L. A. (2015). Versiones en español del Mini mental state examination (MMSE). Cuestiones para su uso en la práctica clínica. Revista Neorología(61 (8:363371)).

Mora Villalobos, L., Contreras Rojas, J., Aguilar, D. V., Henriette, R., Schnaider, B. M., \& Silverman, J. (2017). Desempeño de la prueba "Mini-Mental State Examination" en personas adultas mayores sin deterioro cognitivo. UCR, 24.

Pirani, Z., Bulakiwal, F., Kagalwala, M., \& Kalolwala, M. (2016). Android Based Assistive Toolkit For Alzheimer. ScienceDirect, 9.

Polzer, N., \& Gewald, H. (2017). Smartphone Applications to Early Diagnose Alzheimer 's Disease or Dementia Diagnose. ScienceDirect, 6.

Rodríguez, J. L., \& Gutiérrez Herrera, R. F. (2014). Demencias y enfermedad de Alzheimer en América Latina y el Caribe. Revista Cubana de Salud Pública.

S., P. R. (2010). Ingeniería de software un enfoque práctico. México: Mc Graw Hill.

Violetta, C., \& Susana, M. (2019). La alzheimerización de la vejez. Aportes de una etnografía de los cuidados. Desacatos(59), 130-147. 